\title{
Main Characteristics and Anthropometrics of People with Down Syndrome - Impact in Garment Design
}

\author{
Rochelne Barboza ${ }^{1(\bowtie)}$, Miguel Carvalho ${ }^{1}$, Fernando Ferreira ${ }^{1}$, and Bugao $\mathrm{Xu}^{2}$ \\ 1 Textile Engineering Department, School of Engineering, University of Minho, \\ Campus Azurem, 4800-058 Guimaraes, Portugal \\ chelnebmg@gmail.com, \{migcar, fnunes\}@det.uminho.pt \\ ${ }^{2}$ University of North Texas, Denton, TX, USA \\ Bugao.Xu@unt.edu
}

\begin{abstract}
Among the human chromosome abnormalities, Down Syndrome is the most prominent. Social perception challenges include prejudice, myth and exclusion, with social inclusion having been subject of several studies. From this perspective, the main objective of this study is to contribute to a higher social inclusion of people with Down Syndrome. This is addressed by an anthropometric characterization study of Down Syndrome individuals, performed with the technology of body scanning (3D Body Scanner). The presented study can support the development of inclusive clothing, adapted to people with special needs, promoting the anthropometric and ergonomic aspects of shape, comfort and aesthetics, which would lead to an increased quality of life, self-esteem and security, contributing to a higher inclusion in our society. The results from the data obtained through the measuring tables provided by the 3D Body Scanner System allow the identification of the main body shapes of the analyzed sample, as well as the main variables of their measurements. The impact characteristics from this specific population in the garment design process is also discussed.
\end{abstract}

Keywords: Down Syndrome $\cdot$ Inclusive clothing $\cdot$ Ergonomics $\cdot$ Anthropometry · 3D body scanner

\section{Introduction}

Individuals with genetic alterations, specific physical characteristics, and people with disabilities constitute a considerable population segment in society. It is estimated that the worldwide incidence of people with Down Syndrome or Trisomy 21 (additional copy of chromosome 21) appears at a frequency of 1 to 1100 live births, representing about 3 to 5 thousand children born per year.

The objective of this study is to carry out anthropometric measurements using 3D body scanner technology, seeking to identify within the sample, the main biotypes of people with Down Syndrome and to produce standard size tables with the most relevant measurements for the pattern design process, contributing to a greater social inclusion. Furthermore, this guarantees credibility to individuals, ensuring their integration in the social environment, constructing positive images for themselves and for the World. 
Introducing clothing to be considered an encouraging object and affirming ally of the individual identity, since human beings are complex and subjective. And in the case of disadvantages of a disability, in general, any difference may cause higher impacts.

For Barbosa [1] individuals consume not only for the satisfaction needs, but also for the emulation of others, associating in the search of pleasure, for the defense and affirmation of social status, and so on. According to this author, product consumption is a way to deal with phenomenological insecurity, as the consumer participates in a mutual aid group in order to overcome "mental blockage" and "inhibitions", and thus expressing more intense feelings.

The need for anthropometric studies of the general population is a priority within many countries. Several international research studies are currently under development. However, those who are not included in the target population of such studies, due to different reasons, don't have their needs addressed. People with different types of disabilities take part in such niches research. The need for anthropometric and ergonomic studies, which allow the development of appropriate pattern design, considering specific body shapes and measurements, is a challenge for the scientific community. This study aims to make a contribution.

This study on the corporeal pattern of people with Down Syndrome was conceived, considering aspects like personal fulfillment, self-esteem, equality values within society. The aim is to identify physical characteristics of such individuals and to compare the anthropometric differences amongst people with and without this type of disability. Its main goal is to solve dissatisfaction related to the clothing, to reveal the relevant differences. Furthermore, it aims to develop a specific base model, to support the development of a methodology or guide for the design of clothing adapted to this segment of population needs.

Anthropometry, ergonomics, and comfort are inextricably linked with the human body and clothing domains. When wearing a garment, each body movement performed should engender a positive interaction and provide, although unconsciously, physical, thermophysiological, psychological and social satisfaction, primordial aspects for the balance between body and clothing. In this way, this study will contribute to a greater social inclusion of individuals that are not commonly seen as a market research segment of the fashion industry.

\section{State of the Art}

Regarding anthropometry, during the last decades, some studies have been developed with Down Syndrome individuals. Mainly, these studies aim to obtain information concerning the analysis of related variables, more specifically of body weight, height and skinfolds. However, in such studies, variables are usually isolated from each other. There are few studies that address these variables together, probably due to the operational difficulties in collecting data from this population group.

Other studies address comparative parameters between the standard height curves of people with Down Syndrome with people's height limits by region, justified by the author as: "the definition and design of growth patterns requires accurate methods of 
measurement and reliable, representative registration systems of a given population, considering the appropriate conditions, and distinguishing the biological differences of each population" [2].

The growth curves of people with Down syndrome, from childhood to adulthood, also provided researchers interest on this field. It is assumed that the mean height of Down syndrome individuals in adulthood is reduced by approximately two standard deviations [3].

Nutritional conditions of Down Syndrome people are also the focus of many studies. Normally, such studies apply anthropometric data to establish a proper relationship of weight, height and body structure. Anthropometric methods, due to their ease of execution and low cost, have been the most widely used for nutritional population diagnosis, especially in childhood [4].

In the field of Physical Education, studies focused on general health of Down Syndrome people are identified as the most motivated and validated. Topics such as obesity and body composition assessment gained more attention. However, the number of genes, markers, and chromosomal regions associated or related to human obesity phenotypes is greater than 200 [5].

Psychology is one of the scientific areas that has developed several research regarding the Down Syndrome, mainly addressing the term self-perception. The search for a conceptual definition about self-perception denotes a certain difficulty. Although there is a consensus among authors about its broad meaning, which relates to the premise that self-perception is a set of perceptions that each one has about him/herself. In the case of a mental disabilities population, independent of the level of disability, the understanding of these self-perceptions in their totality and their importance in the behavioral treatment, this theme is of to an extreme significance. One of these studies adapted the Pictorial Scale of Perceived Competence and Social Acceptance for Children with Cerebral Palsy to the Portuguese reality, and was based on the Pictorial Scale of Perceived Competence and Social Acceptance in Young Children [7]. However, research studies with a focus on self-perceptions are limited, as stated by Campos [6].

In some studies, cited by Nunes and Ferreira [8], authors investigate the conceptual approaches of mental disabilities, and the consensus is a need for further discussion among experts, as they believe that the special features of this group are important but also complex. The disabled people, becoming inactive, unproductive and not accepted in society may represent a consequence and not only a cause. Disabled people who live under restrictions and limitations that could be overcome must be constantly stimulated by people from their social circles, by the very environment in which they live, and cultural interests. This stimulate them to develop a greater positivity and a sense of belonging, overcoming difficulties imposed by the society.

Maestrello, quoted by Nunes and Ferreira [8], emphasizes that in recent years, the great emphasis remains on the social role of the mentally disabled individuals and on the conditions that allow their adjustment to the community. A person with a mental disability "is no longer seen as requiring special care, but like a member of the community as a whole." 
Individual skills and their singularities are considered within an adaptive behavior. So, it is necessary to evaluate the various strands of adaptive behavior, which is described as "the efficiency or degree to which an individual meets the standards of personal independence and social responsibility expected for his or her age and social group" [8].

According to the American Association on Mental Retardation (AAMR), areas such as communication, health, safety, leisure, work, academic life, personal area and social skills are of intense relevance for people with Down Syndrome regarding their adaptive behavior. In addition, the progressive evaluation of individuals must be continuous and dynamic, and the professionals involved should act through systematic observations.

The lack of an anthropometric database of Down Syndrome people restricts the development of studies, whether in the field of science, design, or other areas. The available anthropometric data are specific to a given population since they present particular characteristics and determinants related to the environment. Many countries sought to collect anthropometric data as well as the body shape of their consumers, but with people without disabilities. USA, United Kingdom, Germany and French National Dimensioning Campaign are some more recent examples [9]. There is a need to define anthropometric references for people with Down Syndrome and to generate a standardization of measurement tables, patterns size, and statistical database. The mapping of the different biotypes of this specific population is a positive step to meet such need.

\section{Experimental Procedure}

\subsection{Methods and Procedures}

The 3D Body Scanner KBI-Kinect Body Imaging (University of North Texas, Denton, Texas, USA) employed in this study, is a three-dimensional scanning system able to capture the 3D surface of an entire body through four Microsoft Kinect sensors. Two versions of this technology are available: Kinect for Windows and Kinect for Xbox. Although both can be used in the KBI system, the Kinect technology that provides greater precision images, which is due to its speed scanning, is its version for Windows.

The KBI system uses four Kinect sensors, which allow both front and back full body scanning. The sensors are vertically set up, two in front of the individual and two at back, at an approximate distance of $2.6 \mathrm{~m}$. Depending on the height of the individual, this distance may change. If it is not possible to cover the full height of a participant, the distance between the pairs of sensors must be increased.

The static images are captured in a quarter of a second. A measurement error of 2 to $3 \mathrm{~mm}$ in depth can occur if the distance between the individual and the scanner does not match the indicated parameters or the Kinect infrared camera sensor is not properly calibrated. System calibration is critical to measurement accuracy and must be done whenever a data collection is initiated or the sensors are moved by someone. The calibration process involves a special target, located between the two pairs of sensors on the front and back sides, according to the KBI-Kinect Body Imaging User's Manual. 
The KBI - Kinect Body Imaging software organizes the sensors into Groups. Each pair of sensors settled on the same side constitutes a Group. A possible grouping is, for example, Group 1 composed by devices 1 and 3; Group 2 composed by devices 2 and 4. The Group setup and the distance to the ground of each sensor are shown in Fig. 1.

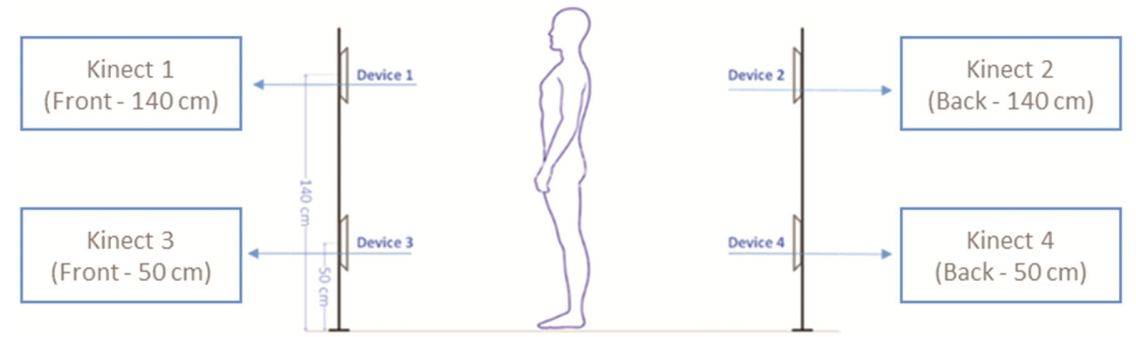

Fig. 1. Sensors set up in the KBI - Kinect Body Imaging.

After grouping the sensors, the system calibration should be done. By using an easyto-detect Target, it is possible to determine the $\mathrm{X} / \mathrm{Y}$, and $\mathrm{Z}$ coordinates which allow the sensors to perform an accurate readout for system calibration. A metallic frame, which has a flat surface, a frontal face parallel to the back face is used in the construction of the Target point. The frame helps in obtaining a precise measurement capture.

This calibration process is required only once before initiating images scanning, except when the equipment is moved. The next step is to update the $\mathrm{X}$, Y, and $\mathrm{Z}$ coordinates for each of the four sensors in the configuration file of the KBI software.

The X, Y and $\mathrm{Z}$ coordinates of each of the 8 corners for the Group 1 and Group 2 sensors must be defined and included in the system calibration file. The $Y$ axis traces the height, the $\mathrm{X}$ axis traces the width, and the $\mathrm{Z}$ axis traces the depth (Fig. 2).

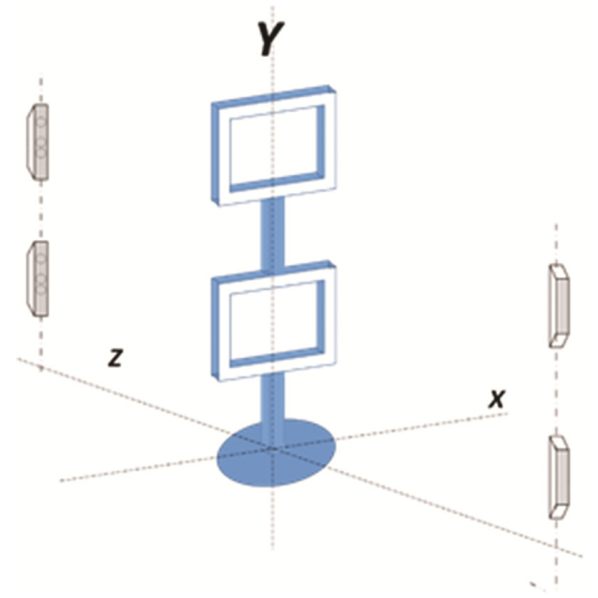

Fig. 2. Geometric Target and $\mathrm{X} / \mathrm{Y} / \mathrm{Z}$ coordinates. 
After a successful calibration, all tridimensional mapping metrics are set and automatically saved in the program folder.

From this time on it is possible to capture images for each individual, which are shown in a new file window.

After each image capture, the next step is saving it on the folder and opening the $3 D$ Body Software, which allows visualization and manipulation of the three-dimensional images. Subsequently, images need to be sharpened and treated. Concluding the afore mentioned protocols provides system processing ready to automatically provide all measured values and volumes of the scanned body (Fig. 3).

\section{D Body}

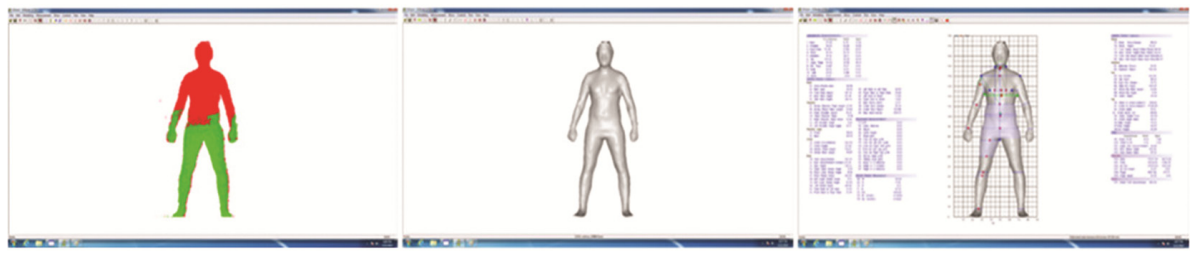

Fig. 3. Image software stages: data cloud image, reconstructed surface image, image with measurements $-3 \mathrm{D}$ body scanner software

\subsection{Sample}

The sample selection follows a purposive sampling, with all participants being Down Syndrome individuals resident in Portugal. To select the participants, contacts with the Portuguese Association of Parents and Friends of Disabled Citizen (APPACDM), located in the municipality of Braga, and the Cooperative for Education and Rehabilitation of Inadvertent Citizens of the Municipality of Guimaraes (CERCIGUI) were established.

The first measurements were accomplished at CERCIGUI during the months of January and February of 2016. In this association, 32 participants with Down's Syndrome were measured, of which 14 males and 18 females, aged from 19 to 47 years old. Subsequently, the results of these samples were availed on an initial pre-test phase. The pre-test phase was crucial to make adjustments of the equipment, for detection of inefficient body postures, and to note the failures and difficulties faced. The understanding of the measurement steps by all persons involved is of paramount importance for the data collection success.

Between the months of May and June 2016, measurements were resumed at APPACDM in Braga municipality. Twenty-eight new individuals were measured, grouped accordingly to gender and age, comprising 15 male and 13 female, from 25 to 50 years old. 


\section{Results and Discussion}

\subsection{D Images Generated by the KBI 3D Body Scanner}

Individually, each participant was requested to remain in underwear clothing while the image was captured. Image capture was repeated more than four times aiming to obtain a more accurate mean measurement and to decrease the standard deviation (Fig. 4).

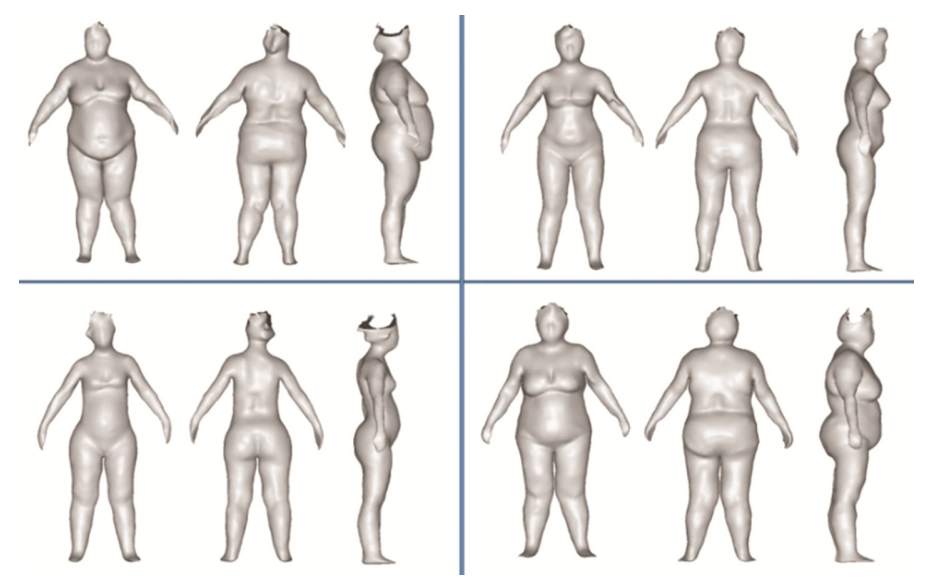

Fig. 4. 3D Body scanner images from female individuals of APPACDM.

\subsection{Comparative Body Analysis}

From the measurement collection and body virtual images obtained through the 3D body scanner, qualitative and quantitative approaches were used for data analysis. Visual analysis was linked with measurements of the variables chosen in the study (bust, waist, hip and abdomen). Also, a correlation among the bust/waist ratio, and hip/waist ratio was enabled to classify the body forms of the individuals of the sample. This classification was based on studies of Karla Simmons [10] who developed a methodology for identifying the types of American women's bodies and provided the basis for the development of the Female Figure Identification (FFIT) software for clothing.

In a first analysis phase, a comparison of the bust measurements with the mean among the waist, abdomen, and hip measurements was done. This procedure allowed to point out the Oval body shape (Bust > Mid Waist/Abdomen/Hip) or Diamond body shape (Bust $<$ Mid Waist/Abdomen/Hip). The calculation of the ratio between bust and waist, and also the ratio between hip and waist in some cases, with a small difference (slightly above $1 \mathrm{~cm}$ ) showed the Hourglass body shape. Cases in which there was a more expressive difference in the hip region, the Inferior Hourglass body shape was identified. When there was a considerable difference between the measurements of the girth of the bust and hip, the Spoon body shape was identified. Another parameter used was the waist line, which has no mark for the Triangle body shape. 
It is important to remark that the studied population is considered mostly Endomorphic, because they have rounded forms, with considerable deposits of fat tissue, prominent abdomen, short legs, and short arms [11].

According with the geometric forms and FFIT classifications [12], comparative analyses of the different body shapes found in the sample study were made, supported by the 3D body scanning system - KBI.

As mentioned, sampling groups were created according to the ages, enabling a further analysis of their possible differences. Group 1 was comprised of individuals between the ages of 21 and 30, Group 2 was comprised of individuals between the ages of 31 and 40, and Group 3 of individuals between the ages of 41 and 50 years old (Figs. 5 and 6).

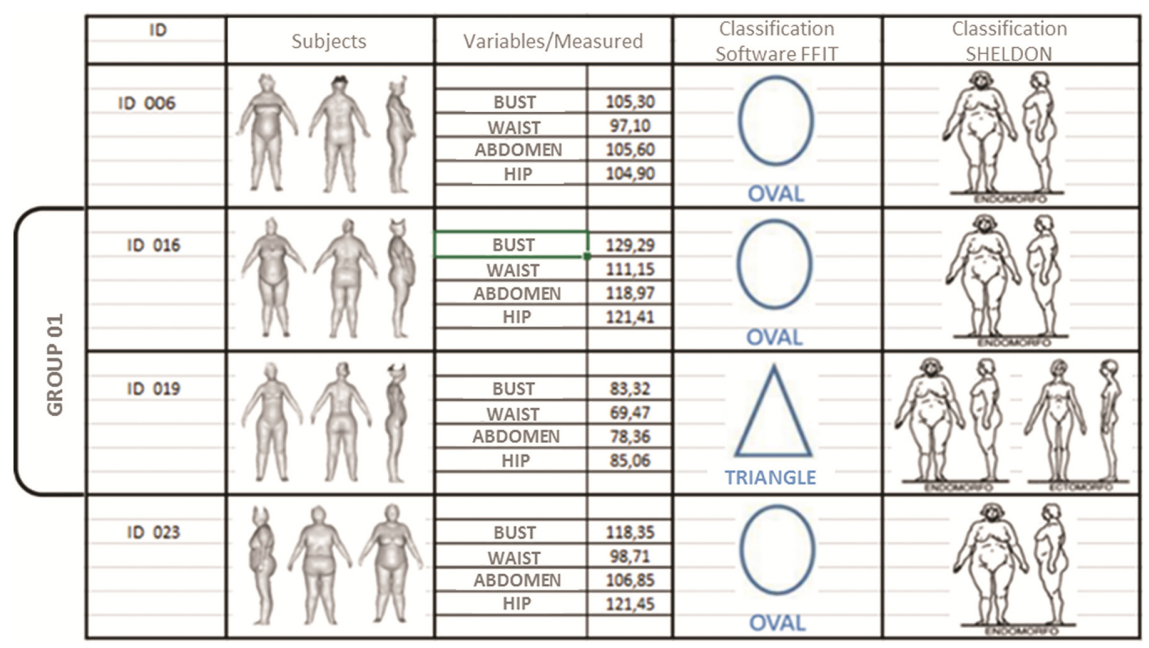

Fig. 5. Comparison among the main biotypes and the study target population.

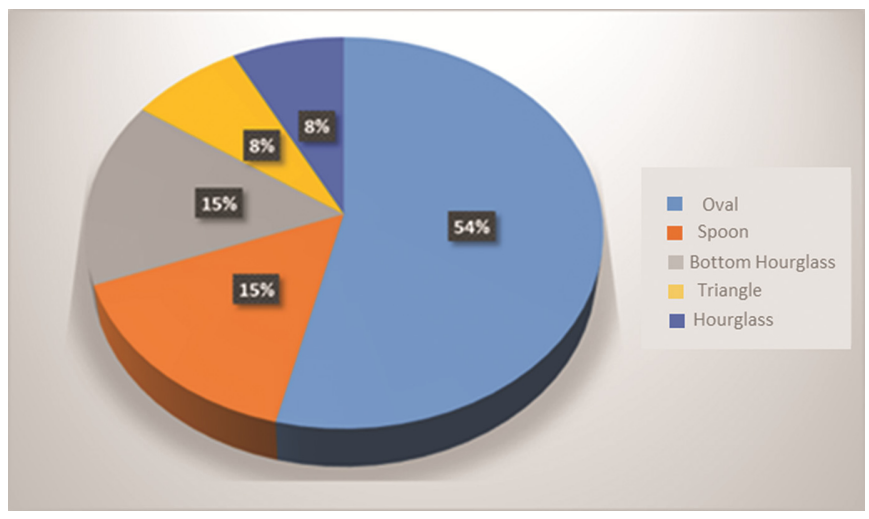

Fig. 6. Body shapes of the female Down Syndrome sample. 


\subsection{Table Measurements Results}

A Microsoft Excel spreadsheet containing the measurement data of each participant was created. Only 33 body measurements (including height) were selected, and weight and age were also added to the spreadsheet. The measurements were selected in order to allow the development of the pattern design methodology and to achieve the study goals (Table 1).

Table 1. Female measurements - APPACDM (Portugal) participants

\begin{tabular}{|c|c|c|c|c|c|c|c|c|c|c|c|c|c|c|}
\hline Ordem & & ID002 & ID006 & ID007 & ID008 & ID009 & ID010 & ID011 & ID014 & ID015 & ID016 & ID019 & ID020 & ID023 \\
\hline & Age & 42 & 30 & 38 & 37 & 34 & 48 & 34 & 34 & 32 & 25 & 27 & 35 & 30 \\
\hline & Weight & 45,000 & 57,600 & 78,100 & 60,400 & 65,000 & 77,300 & 77,800 & 61,900 & 62,400 & 93,000 & 46,600 & 54,600 & 74,100 \\
\hline 1 & 1. Neck circumference & 41,92 & 42,62 & 42,68 & 43,43 & 43,76 & 41,84 & 41,59 & 43,14 & 37,72 & 47,69 & 34,61 & 43,23 & 43,71 \\
\hline 2 & 2. Shoulder width & 34,30 & 37,64 & 41,56 & 40,91 & 42,43 & 42,37 & 38,33 & 39,34 & 41,20 & 42,23 & 33,32 & 41,14 & 41,31 \\
\hline 3 & 2. Shoulder depth & 14,59 & 16,10 & 14,10 & 15,70 & 15,89 & 17,21 & 14,41 & 18,39 & 14,19 & 19,35 & 16,23 & 16,52 & 16,97 \\
\hline 4 & 3. Bust circumference & 84,76 & 105,25 & 108,84 & 107,33 & 113,93 & 109,74 & 100,71 & 100,19 & 105,02 & 129,29 & 83,32 & 95,69 & 118,35 \\
\hline 5 & 4. Waist circumference & 73,00 & 97,10 & 92,89 & 93,96 & 99,78 & 93,97 & 86,49 & 85,34 & 89,62 & 111,15 & 69,47 & 75,71 & 98,71 \\
\hline 6 & 5. Abdomen circumference & 84,45 & 105,56 & 104,34 & 106,44 & 106,90 & 120,58 & 98,04 & 96,88 & 101,48 & 118,97 & 78,36 & 89,99 & 106,85 \\
\hline 7 & 6. Hip circumference & 100,39 & 104,86 & 111,10 & 112,15 & 115,06 & 128,70 & 117,87 & 109,66 & 104,82 & 121,41 & 85,06 & 99,59 & 121,45 \\
\hline 8 & 7. Upper thigh circumference & 62,41 & 58,00 & 72,46 & 64,04 & 57,18 & 72,83 & 71,00 & 66,14 & 60,50 & 70,85 & 59,92 & 60,07 & 68,07 \\
\hline 9 & 8. Mid thigh circumference & 48,60 & 46,14 & 57,21 & 48,82 & 46,69 & 60,05 & 63,54 & 51,02 & 48,67 & 59,26 & 48,38 & 49,98 & 54,53 \\
\hline 10 & 9.Knee circumference & 34,72 & 35,02 & 42,55 & 35,08 & 32,20 & 42,51 & 45,40 & 37,86 & 35,34 & 41,71 & 40,20 & 38,36 & 45,92 \\
\hline 11 & 10. Calf girht circumference & 36,44 & 37,16 & 43,19 & 36,82 & 37,22 & 41,95 & 43,21 & 39,88 & 37,71 & 45,28 & 40,43 & 40,34 & 46,85 \\
\hline 12 & 11. Ankle circumference & 22,20 & 23,57 & 26,88 & 24,07 & 23,20 & 28,19 & 29,78 & 27,15 & 23,78 & 27,34 & 28,48 & 27,11 & 29,27 \\
\hline 13 & 13. Base neck & 32,18 & 39,72 & 31,64 & 37,71 & 32,25 & 38,22 & 34,01 & 34,45 & 34,14 & 39,58 & 34,32 & 33,98 & 35,96 \\
\hline 14 & 14. Front neck height & 109,99 & 109,52 & 121,07 & 107,64 & 111,18 & 118,96 & 130,58 & 117,19 & 118,88 & 123,49 & 114,17 & 114,17 & 115,28 \\
\hline 15 & 15. Back neck height & 113,73 & 113,47 & 125,07 & 111,65 & 115,35 & 124,01 & 134,06 & 121,30 & 122,24 & 127,99 & 117,48 & 118,32 & 120,00 \\
\hline 15 & 17. Across shoulder front length & 19,89 & 20,04 & 23,26 & 21,43 & 22,70 & 25,16 & 27,11 & 23,61 & 22,15 & 25,22 & 17,70 & 23,25 & 24,53 \\
\hline 17 & 18. Across shoulder back length & 22,86 & 23,18 & 23,63 & 23,28 & 24,15 & 26,31 & 26,18 & 25,03 & 24,13 & 26,41 & 20,84 & 24,55 & 25,99 \\
\hline 18 & 32. Bust height & 93,53 & 90,27 & 102,56 & 91,80 & 95,46 & 100,74 & 110,59 & 96,86 & 100,32 & 103,32 & 98,81 & 97,71 & 96,40 \\
\hline 19 & 41. Front neck to left bust & 18,22 & 23,11 & 23,26 & 19,24 & 20,71 & 21,45 & 23,71 & 22,76 & 22,85 & 27,07 & 17,57 & 19,56 & 21,89 \\
\hline 20 & 42. Front neck to right bust & 18,79 & 23,33 & 22,40 & 18,97 & 19,40 & 22,05 & 24,22 & 23,16 & 23,26 & 27,85 & 17,25 & 20,16 & 22,20 \\
\hline 21 & 76. Waist height & 83,33 & 77,30 & 96,22 & 79,71 & 86,06 & 88,91 & 101,39 & 89,59 & 91,38 & 93,56 & 86,52 & 88,56 & 87,82 \\
\hline 22 & 79. Front full length (neck-bust-waist) & 32,86 & 43,06 & 33,81 & 37,87 & 33,61 & 40,83 & 38,34 & 38,43 & 38,25 & 41,83 & 33,92 & 35,92 & 37,18 \\
\hline 23 & 80. Back full length (neck-bust-waist) & 35,87 & 39,71 & 33,39 & 37,86 & 36,87 & 40,08 & 36,12 & 36,09 & 35,14 & 38,84 & 35,35 & 34,96 & 37,64 \\
\hline 24 & 84. Hip Height & 64,71 & 66,25 & 82,32 & 68,01 & 67,55 & 71,61 & 75,92 & 69,70 & 81,63 & 83,63 & 76,02 & 69,76 & 70,55 \\
\hline 25 & 89. Crotch height & 56,06 & 50,90 & 62,41 & 54,41 & 53,46 & 60,73 & 67,47 & 59,40 & 61,79 & 62,22 & 56,74 & 59,09 & 57,74 \\
\hline 26 & 94. Croth length-front & 38,34 & 37,98 & 48,67 & 27,44 & 39,49 & 46,36 & 46,65 & 41,60 & 40,71 & 33,40 & 36,72 & 37,22 & 46,37 \\
\hline 27 & 95. Crotch length-back & 35,29 & 35,03 & 46,81 & 37,18 & 54,80 & 45,59 & 45,42 & 42,52 & 38,34 & 61,54 & 39,00 & 38,19 & 40,21 \\
\hline 28 & 93. Crotch length full & 73,63 & 73,01 & 95,47 & 64,62 & 94,29 & 89,03 & 92,07 & 84,12 & 79,04 & 94,94 & 75,73 & 75,75 & 86,58 \\
\hline 29 & 96. Thigh height & 55,42 & 50,41 & 62,08 & 53,71 & 52,36 & 59,89 & 67,25 & 58,71 & 61,23 & 59,80 & 56,65 & 58,66 & 57,47 \\
\hline 30 & 99. Elbow circumference & 22,29 & 24,99 & 30,31 & 28,27 & 25,60 & 28,02 & 25,91 & 26,89 & 23,30 & 30,10 & 22,57 & 24,61 & 26,39 \\
\hline 31 & 100. Wrist circumference & 15,24 & 16,71 & 21,33 & 21,54 & 18,40 & 16,74 & 16,34 & 15,30 & 14,18 & 16,70 & 15,93 & 12,97 & 15,78 \\
\hline 32 & 101. Upper arm circumference (bicep) & 27,77 & 27,52 & 35,84 & 30,87 & 30,87 & 41,83 & 29,10 & 42,52 & 36,92 & 48,15 & 30,53 & 40,14 & 42,36 \\
\hline 33 & Height & 1,31 & 1,34 & 1,46 & 1,32 & 1,34 & 1,43 & 1,56 & 1,42 & 1,44 & 1,48 & 1,39 & 1,40 & 1,39 \\
\hline
\end{tabular}

\subsection{Comparative Graphs}

In the following graphs, comparisons between healthy individuals, considered as within the normality, of $1.65 \mathrm{~m}$ of height (measurement of a standard Portuguese table) and male individuals with Down syndrome participants in the study are presented (Fig. 7). 


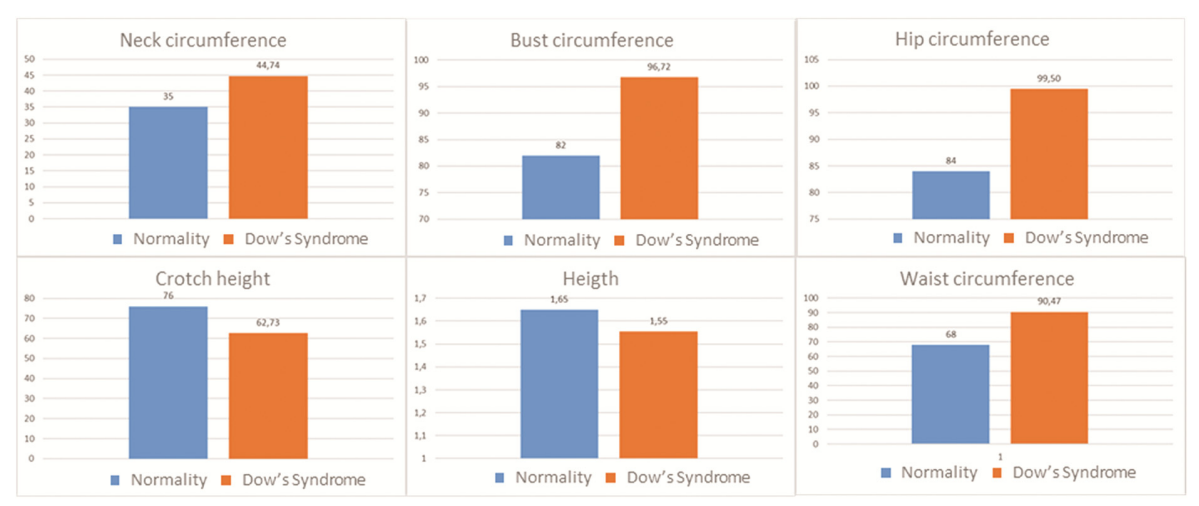

Fig. 7. Comparative graphs between Down Syndrome and healthy male population.

\section{Conclusion}

The bibliographical review and the comparison of the anthropometric data collected allowed a detailed analysis of the body shapes and enabled the identification of the dominant biotypes of the participants, which provided a knowledge enlargement regarding the study topic.

From the 105 measurements that the KBI system provides automatically, 32 measurements were selected. These were identified as having the greatest impact on the development of flat pattern design methodology representative in terms of the characteristics of the standard body of Down Syndrome people.

The analysis of the body shapes was performed in order to distinguish them according to the main biotypes. Furthermore, it was possible to identify a very typical pattern.

The comparison among the measurement results between non-disabled people and the Down's Syndrome people involved in this study, confirmed the existence of very significant differences, which has a great impact in an appropriate pattern design methodology to this population group segment. It is possible to highlight: significant differences between the length of the lower and upper limbs, with measurements lower than the mean values, which is one of the most relevant characteristics of the person with Down Syndrome; larger neck circumference diameter values and short length neck; larger upper body dimensions and less difference among waist, abdomen and hip circumferences measurements, which justifies the predominance of the Oval body shape. Regarding the height, both the female and the male individuals of the sample are shorter than the non-disabled person height average.

There were no significant differences in measurements between participants of the same gender and of different ages, meaning that grouping individuals according to their age is unnecessary in this case.

The bodies of Down Syndrome people have particular characteristics which traditional garment pattern design and assembly processes do not fulfill. However, these individuals seek for the same comfort, in all its features, the same design, the same 
suitability and plurality related to the sense of wearing. The feeling of belonging, the appropriate addressing of their needs, and the same visibility regarding the importance in creating a sense of being are also pursued. These are goals that face many rules, labels, and attitudes that push Down Syndrome people to marginalized positions.

Acknowledgments. This work is financed by FEDER funds through the Competitive Factors Operational Program (COMPETE) POCI-01-0145-FEDER-007136 and by national funds through FCT-Portuguese Foundation for Science and Technology, under the project UID/CTM/ 000264 .

\section{References}

1. Barbosa, L., Campbell, C.: Cultura, consumo e identidade. FGV Editora, Rio de Janeiro (2006)

2. Hall, J.G., Froster-Iskenius, U.G., Allanson, J.E.: Handbook of Normal Physical Measurements. Oxford University Press, New York (1995)

3. Mustacchi, Z.: Curvas padrão pôndero-estatural de portadores de Síndrome de Down procedentes da região urbana da cidade de São Paulo. Doctoral thesis, Universidade de São Paulo, São Paulo (2002)

4. Sarni, R.S.: Avaliação da condição nutricional de crianças e adolescentes-método antropométrico. Temas de nutrição em pediatria 2, 28-38 (2001)

5. Chagnon, Y.C., Pérusse, L., Weisnagel, S.J., Rankinen, T., Bouchard, C.: The human obesity gene map: the 1999 update. Obes. Res. 8, 89-117 (2000)

6. Campos, M.J.C.: Autopercepções em crianças e jovens com síndrome de Down: estudo da competência percebida e da aceitação social. Doctoral thesis, Universidade do Porto, Porto (2005)

7. Harter, S., Pike, R.: The pictorial scale of perceived competence and social acceptance for young children. Child Dev. 55, 1969-1982 (1984)

8. Nunes, L.R.O.P., Ferreira, J.R.: Deficiência Mental: O Que as Pesquisas Brasileiras tem Revelado. Tendências e Desafios da Educação Especial, pp. 51-81. SEESP, Brasilia (1994)

9. Vuruskan, A., Bulgun, E.: Identification of female body shapes based on numerical evaluations. Int. J. Clothing Sci. Technol. 23, 46-60 (2011)

10. Simmons, K.P.: Body shape analysis using three-dimensional body scanning technology. Doctoral thesis, North Carolina State University, Raleigh (2003)

11. Sheldon, W.H., Stevens, S.S., Tucker, W.B.: The Varieties of Human Physique: An Introduction to Constitutional Psychology. Harper, New York (1940)

12. Istook, L.C., Simmons, K.P., Devarajan, P.: Female figure identification technique (FFIT) for apparel. In: Proceedings of the International Conference on Fashion and Textiles (2002) 